\title{
A Influência do Estresse na Condição de Afastamento do Trabalho por Distúrbios Osteomusculares 1
}

Stress Influence on Sick Leave by Musculoskeletal Disorders

\section{A Influência do Estresse na Condição de Afastamento do Trabalho}

\author{
Paulo Eduardo Benzoni2
}

\begin{abstract}
Resumo
Os distúrbios osteomusculares constituem uma das principais causas de licença-saúde pelo INSS e o estresse tende a intensificar tais distúrbios, gerando mais tempo de afastamento e dificuldades de reinserção desses trabalhadores no mercado de trabalho. O objetivo deste estudo foi analisar a influência do estresse na percepção da condição de afastamento do trabalho. Participaram 116 trabalhadores do setor privado, afastados do trabalho por mais de 30 dias corridos, devido a problemas osteomusculares. Avaliou-se o estresse pelo Inventário de Sintomas de Stress de Lipp e a percepção da condição de afastamento pelo Inventário de Percepção de Afastamento do Trabalho de Benzoni e Bragion. Os resultados mostraram uma prevalência de 69\% de estresse nos pesquisados e uma percepção mais negativa da condição de afastamento nos participantes com estresse. Os dados apontaram que o estresse apresenta-se comórbido aos problemas osteomusculares, podendo dificultar a recuperação e reinserção social do trabalhador afastado no mercado.
\end{abstract}

Palavras-chave: Afastamento do trabalho. Estresse. Percepção social.

\begin{abstract}
The musculoskeletal disorders are the main cause of sick leave in INSS in Brazil and, the stress can intensify these disorders increasing the time of sick leave and hindering the return to work of these employees. The objective of this work was to analyze the stress influence in the sick leave perception. 116 employees from private sector on sick leave for more than 30 days participated. The stress was evaluated by the Stress Symptoms Inventory by Lipp and the perception of sick leave condition by the Perception of Sick Leave Inventory by Benzoni and Bragion. The results showed a prevalence of $69 \%$ of stress in the participants and a more negative perception of sick leave condition in participants with stress. Data pointed that stress is comorbid on musculoskeletal disorders and can make recovery and return to work more difficult.
\end{abstract}

Keywords: Sick leave. Stress. Social perception.

\footnotetext{
${ }^{1}$ Pesquisa financiada pela Vice Reitoria de Pós-Graduação e Pesquisa da UNIP - Universidade Paulista através do Programa Individual de Pesquisa para Docentes.

${ }^{2}$ Universidade Paulista (Unip), São Paulo, Brasil. E-mail: paulobenzoni@uol.com.br
} 


\section{Introdução}

Estar trabalhando, ter uma ocupação ativa, corresponde a uma das principais referências psicossociais do ser humano moderno. A ocupação ativa confere ao indivíduo um status social, posicionando-o em algum lugar específico na teia da sociedade de consumo. Sendo assim, o que acontece quando, forçosamente, o trabalhador se vê incapacitado fisicamente de exercer seu trabalho e de se posicionar socialmente, tendo que se afastar da atividade laboral por motivo de saúde?

A Organização Internacional do Trabalho - OIT (1989) denomina o afastamento do trabalho como um quadro incapacitante que leva a uma licença compulsória por doença, impossibilitando, total ou parcialmente, o trabalhador de permanecer com suas atividades profissionais. Por vezes, o quadro incapacitante pode não levar ao afastamento em si, mas gerar situações de absenteísmo marcadas por faltas e atrasos ao trabalho.

No Brasil, dados do Instituto Nacional de Seguro Social (INSS) demonstram que os afastamentos por distúrbios osteomusculares e do tecido conjuntivo (CID $10-\mathrm{M}$ ) corresponderam em 2014 à primeira causa de afastamentos por licença-saúde e, de um total de 1.090 .889 auxíliosdoença ativos nas regiões urbanas, 318.002 correspondiam aos afastamentos por Doenças do Sistema Osteomuscular e do Tecido Conjuntivo com auxílios-doença ativos (Ministério da Previdência Social, 2014).

Ao se considerar o compulsório afastamento do trabalho por períodos superiores a 30 dias consecutivos, em função de uma lesão incapacitante do corpo, pode-se notar a geração de inúmeros problemas ao indivíduo, uma vez que essa condição de afastamento o retira da posição que ocupa na teia social e pode desencadear problemas de ordem psicossocial. Nesse sentido, Boschco (2011) pontua que a situação do afastamento do trabalho implica em múltiplas repercussões psicossociais aos indivíduos envolvidos, visto que a doença traz consequências não somente em relação ao trabalho, mas também em aspectos do cotidiano, nas relações interpessoais, causando sentimentos de exclusão, insegurança e angústia. Na mesma linha, Ramos, Titoni e Nardi (2008) pontuam que o trabalhador afastado carrega um sentimento de ter falhado, de fragilidade e inferioridade em comparação aos seus pares e superiores, por não conseguir responder adequadamente às exigências produtivas. Tais sentimentos negativos sobre si mesmo em relação à capacidade produtiva, em conjunto com o sentimento de incapacidade laboral, pode levar a uma intensificação do quadro patológico, uma espécie de ganho secundário que a doença geradora do afastamento pode possibilitar (Benzoni, 2012). Estando incapaz para o trabalho, o trabalhador não será obrigado a enfrentar seus medos e angústias e a experiência de fracasso pela impossibilidade de trabalhar, que são apontadas por Souza e Faiman (2007), como acompanhando o processo de retorno ao trabalho, residiria então aí o ganho secundário.

As chamadas Doenças do Sistema Osteomuscular e do Tecido Conjuntivo (CID 10 - M), englobam, entre outras, os Distúrbios Osteomusculares Relacionados ao Trabalho (Dorts), e estes têm sido estudado de forma multidisciplinar, tanto no Brasil como em outros países. Esses estudos apontam para uma origem multicausal dos Dorts, envolvendo aspectos não 
puramente biomecânicos, mas psicológicos e sociais como contribuintes ao seu desenvolvimento (Andrade \& Barbosa-Branco, 2015; Bugaiska et al., 2013; Zakerian \& Subramaniam, 2011; Almeida, Carvalho Filho, Pastre, Lamari \& Pastre, 2008 e National Research Council, 2001). Dentre os fatores psicológicos, pode-se destacar o papel do estresse no Dort. Eatough, Way e Chang (2012) pontuam que altos níveis de conflito de papéis e baixa autonomia no trabalho, fatores esses geradores de tensão e estresse, se relacionam diretamente com altos índices de sintomas osteomusculares.

Tomando todos esses aspectos sobre o trabalhador em condição de afastamento, questiona-se como o estresse pode interferir na recuperação do trabalhador afastado, tanto do ponto de vista físico do distúrbio como social, de posicionamento social e retorno ao trabalho.

Primeiramente descrito por Selye, em 1950, o estresse consiste na interação entre perigo e defesa. Trata-se de uma resposta neuropsicofisiológica do organismo a qualquer evento que possa colocar a integridade física e psicológica do indivíduo em perigo (Selye, 1950 e 1959).

Atrelado a outros fatores, o estresse consiste em uma das principais fontes de sofrimento no trabalho (Antloga, Maia, Cunha \& Peixoto, 2014). Silveira-Maissat, Lautert, Dal Pai e Tavares (2015) consideram que a vivência do esgotamento, que se dá pela percepção de estresse, insatisfação, sobrecarga, frustração, insegurança ou medo é a expressão clara do sofrimento de ordem ocupacional. No contexto de trabalho, os principais agentes causadores e geradores de estresse incluem aspectos de organização, administração, sistemas de trabalho e qualidade das relações humanas. O trabalho no qual a exigência e a pressão superam o conhecimento e a capacidade do trabalhador é o que mais produz estresse, principalmente quando aliado à baixa possibilidade de tomada de decisão, baixa autonomia e pouco apoio dos colegas (Leka, Griffiths \& Cox, 2004).

Existem diferenças interindividuais na reação aos estímulos causadores de estresse que começam a se manifestar pelo grau de impacto causado pelo estressor em cada pessoa. Esse grau é determinado por características do estímulo, pela habilidade que o organismo tem em lidar com a situação e por características do indivíduo, tais como o repertório de enfrentamento que ele possui. Todos esses fatos ocorrem em função de variáveis individuais (sexo, idade, temperamento, fatores relativos à controlabilidade sobre $\mathrm{O}$ estressor e fatores sociais, tais como posto e/ou suporte sociais), fazendo com que alguns indivíduos sejam mais resilientes que outros (Souza, Silva \& Galvão-Coelho, 2015).

Considerando o estresse em função da percepção que o trabalhador afastado tem de sua própria condição psicossocial, e baseando-se nas colocações sobre situação de afastamento, podese tomar o estresse como uma das possíveis expressões concretas, e até certo ponto mensurável, do sofrimento pela incapacidade ao trabalho. Para tanto se faz necessário compreender e explorar o conceito de percepção social.

Berger e Luckmann (1990) dizem que a percepção social compreende um conjunto de valores e crenças que filtram a realidade que circunda o indivíduo, atribuindo-lhe significados e, nesse sentido, consideram a percepção como uma atividade psicológica que deve ser 
interpretada a partir do contexto psicossocial ao qual o indivíduo que percebe se encontra.

Assim, o presente trabalho teve por objetivo analisar a influência do estresse na percepção social que o trabalhador afastado apresenta de sua condição de afastamento.

\section{Método}

\section{Participantes}

Foi utilizada uma amostra não probabilística por acessibilidade composta por 116 trabalhadores afastados do trabalho, por mais de 30 dias, devido a distúrbios osteomusculares e do tecido conjuntivo (CID $10-\mathrm{M}$ ). Todos os participantes foram de uma mesma região no interior do estado de São Paulo e recrutados por meio de três fontes: Indicação (14,7\%), Clínica Escola de Fisioterapia de uma universidade privada $(6,9 \%)$ e o Cerest - Centro de Referência em Saúde do Trabalhador / Ambulatório de Saúde do Trabalhador (78,4\%).

O perfil ocupacional dos participantes foi classificado segundo os Grandes Grupos Ocupacionais da CBO - Classificação Brasileira de Ocupações (Ministério do Trabalho e Emprego, 2010) e concentrou-se em trabalhadores de serviços, vendedores de comércio com 71, $6 \%$, contando também com $12,9 \%$ de pesquisados do setor industrial, $6 \%$ de trabalhadores em serviços administrativos, 3,4\% de técnicos de nível médio, 2,6\% de profissionais das ciências e das artes e 3,5\% de operadores de processos contínuos. A maior parte dos participantes, 65,6\%, foram do setor de serviços, seguido de $18 \%$ comércio e $16,4 \%$ indústria.

No que se refere ao perfil de afastamento, a amostra apresentou uma concentração em afastamentos de até 12 meses
(77,6\%), o restante se distribuiu homogeneamente entre 13 e 31 meses ou mais.

\section{Instrumentos}

Utilizou-se um questionário de caracterização demográfica e social com questões objetivas e, tendo como foco compreender a influência do estresse na condição de afastamento do trabalho, foram utilizados dois instrumentos para coleta de dados.

Para identificação do estresse utilizou-se o ISSL - Inventário de Sintomas de Stress para Adultos de Lipp (Lipp, 2005), um teste padronizado e validado para identificação da presença de estresse que consiste em um teste psicológico recomendado pelo CFP - Conselho Federal de Psicologia.

Utilizou-se, também, o Ipat - Inventário de Percepção de Afastamento do Trabalho de Benzoni e Bragion (2016) para avaliar a percepção da condição de afastamento. O Ipat consiste em um inventário de 22 afirmativas sobre a condição de afastamento, as quais devem ser classificadas em uma escala Likert de 1 a 5, sendo que quanto maior a nota melhor é a percepção do respondente naquele item. O resultado do Ipat possibilita identificar a percepção do afastamento em seis fatores: expectativas de recepção no ambiente de trabalho ao retornar, motivação para retornar ao trabalho, suporte social à condição de afastamento, percepção de apoio familiar, percepção de incapacidade para o trabalho e ser visto como tirando vantagem do afastamento.

\section{Procedimento}

Os dados foram coletados no período de setembro de 2015 a dezembro de 2016 nas instituições parceiras no projeto, bem como por 
indicações. Os critérios éticos documentais de Termo de Consentimento Livre e Esclarecido foram cumpridos, tanto para os participantes como instituições. A aplicação dos instrumentos foi feita individualmente, em sala privativa e, como contrapartida imediata, oferecia-se ao participante uma cartilha ilustrada com conteúdo educativo sobre estresse e formas de controlá-lo.

Para análise dos dados, os resultados de cada instrumento e os dados de caraterização sociodemográficos, foram tratados numericamente utilizando-se o programa IBM SPSS Statistical Package for Social Sciences, versão 21, realizando-se análises por estatística descritiva, testes Qui-quadrado e $t$ de Student.

O projeto foi submetido ao Comitê de Ética da universidade, tendo sido aprovado no mês de abril de 2015 (Parecer no 1.063 .794 de 14/05/2015).

\section{Resultados}

A amostra apresentou um perfil de $41,4 \%$ de homens e $58,6 \%$ de mulheres, o estado civil da amostra apresentou 39,7\% de solteiros e $41,4 \%$ de casados. A faixa etária variou de 18 a 61 anos, com 62,9\% entre 41 e 60 anos. Quanto ao número de filhos, $15,5 \%$ não tinham filhos e 68,1\% tinham entre 1 e 3 filhos.

A escolaridade dos participantes variou de fundamental incompleto $(41,4 \%)$ a superior completo $(4,3 \%)$ e $35,4 \%$ estavam com ensino médio completo ou incompleto.

A prevalência de estresse na amostra como um todo foi de 69,0\%. Observando a distribuição entre sexos, $56,3 \%$ dos homens apresentaram estresse e $77,9 \%$ das mulheres o apresentaram. A distribuição entre os sexos apresentou uma diferença significativa $(p=0,01)$ pelo teste qui-quadrado.

Com relação ao estado civil, não foram observadas concentrações nos subgrupos e a distribuição por faixa etária evidenciou que somente dentro da faixa etária de 21 a 30 anos o percentual de pesquisados sem estresse foi maior $(57,1 \%)$ do que dos que não têm estresse $(42,9 \%)$, nas demais o percentual com estresse foi maior, porém não foram observadas diferenças significativas entre as faixas.

A profissão dos pesquisados foi classificada a partir dos grandes grupos ocupacionais da CBO (Classificação Brasileira de Ocupações) e somente no grupo de trabalhadores nas ciências e nas artes o percentual de com estresse foi menor $(33,3 \%)$ do que o de sem estresse $(66,7 \%)$. Nos demais casos o percentual de com estresse foi maior, sendo técnicos de nível médio 100\%, trabalhadores de serviços administrativos $71,4 \%$ e trabalhadores dos serviços, vendedores do comércio em lojas e mercados $73,5 \%$. Não foram observadas diferenças significativas entre os grupos.

No tocante ao tempo de afastamento, o percentual de trabalhadores afastados com estresse se mostrou maior em todas as faixas, ficando mais evidenciado nas faixas acima de dez meses de afastamento, com um percentual médio de $85 \%$ de trabalhadores com estresse.

Referente às áreas de atuação, houve uma concentração significativa $(p=0,01)$ de trabalhadores afastados com estresse nas áreas de serviços $(78,7 \%)$ e comércio $(61,9 \%)$. 
Tabela 1. Comparação entre as médias dos grupos sem estresse e com estresse no Inventário de Percepção de Afastamento do Trabalho (Ipat)

\begin{tabular}{|c|c|c|c|c|c|}
\hline \multirow{2}{*}{ Fator } & \multicolumn{2}{|c|}{ Sem estresse } & \multicolumn{2}{|c|}{ Com estresse } & \multirow{2}{*}{ Significância } \\
\hline & Média & DP & Média & DP & \\
\hline $\begin{array}{l}\text { F1 - Expectativas de recepção no ambiente } \\
\text { de trabalho ao retornar. }\end{array}$ & 3,91 & 1,15 & 2,95 & 1,30 & $\mathrm{p}=0,01$ \\
\hline F2 - Motivação para retornar ao trabalho. & 3,97 & 1,12 & 3,83 & 1,06 & $\begin{array}{c}\text { Não } \\
\text { Significativa }\end{array}$ \\
\hline $\begin{array}{l}\text { F3 - Suporte social à condição de } \\
\text { afastamento. }\end{array}$ & 4,23 & 1,02 & 3,68 & 1,19 & $\mathrm{p}=0,01$ \\
\hline F4 - Percepção de apoio familiar. & 4,53 & 0,87 & 4,15 & 1,04 & $\mathrm{p}=0,05$ \\
\hline $\begin{array}{l}\text { F5 - Percepção de Incapacidade para o } \\
\text { trabalho. }\end{array}$ & 3,80 & 1,28 & 3,25 & 1,25 & $\mathrm{p}=0,03$ \\
\hline $\begin{array}{l}\text { F6 - Ser visto como tirando vantagem do } \\
\text { afastamento. }\end{array}$ & 2,25 & 1,48 & 2,25 & 1,31 & $\begin{array}{l}\text { Não } \\
\text { Significativa }\end{array}$ \\
\hline
\end{tabular}

Elaborada pelo autor.

$\mathrm{Na}$ avaliação da percepção de afastamento, o índice de confiabilidade (alfa de Cronbach) obtido para o Ipat foi de 0,84. Observando as médias gerais nos fatores avaliados pelo Ipat (Tabela 1), observou-se que o grupo de trabalhadores afastados que apresentam estresse avalia de forma significativamente pior as expectativas de recepção no ambiente de trabalho (F1 p=0,01), o suporte social à condição de afastamento (F2 p=0,01), o apoio familiar que têm $(\mathrm{F} 4 \mathrm{p}=0,05)$ e a percepção de sua capacidade para o trabalho ( $F 5 \mathrm{p}=0,02)$, quando comparados ao grupo de trabalhadores afastados que não apresentam estresse.

As afirmativas do Ipat que apresentaram diferenças mais significativas versaram sobre a questão de como os superiores poderiam perceber a situação de afastamento e como receberiam o trabalhador, quando de seu retorno.

\section{Discussão}

A amostra utilizada demonstrou equilíbrio entre participantes solteiros e casados (41,4\% em cada). Segundo Martinez, Latorre e Fisher (2010), as mulheres tendem a um maior risco de perda da capacidade para o trabalho, o que pode estar associado à condição da mulher na sociedade, exposta a dupla jornada e estressores sociais mais intensos. Nesse sentido, observou-se um leve predomínio de mulheres $(58,6 \%)$ na amostra.

Houve uma concentração na faixa etária acima de 41 anos, o que encontra correlato nos trabalhos de Martins et al. (2009) em levantamentos realizados com trabalhadores afastados na área de saúde, e por Andrade e Barbosa-Branco (2015) em levantamento sobre benefícios concedidos a casos de Sinovite e Tenossinovite no Brasil, esses autores também observaram que essa idade apresentava concentração maior. Martinez et al. (2010) 
pontuam que a partir dos 45 anos a capacidade para o trabalho começa a diminuir devido a aspectos de envelhecimento natural do corpo com consequente perda de força física, o que também contribui para explicar os dados relativos à concentração de faixa etária da amostra.

O perfil ocupacional dos participantes concentrou-se em trabalhadores de serviços, vendedores de comércio com 71,6\%. A cidade, local da coleta dos dados, consiste em um grande centro comercial regional e tem sua economia baseada em comércio e prestação de serviços. Corroborando esse dado, Almeida et al. (2008), avaliando a demanda de casos de afastamento do trabalho por afecção do tendão supraespinhal, identificaram também uma concentração de casos advindos do setor de serviços.

Observou-se uma prevalência considerável de estresse na amostra pesquisada, sendo que $69,0 \%$ dos pesquisados o apresentaram. Esse percentual é considerado elevado se comparado aos dados de padronização do Inventário de Sintomas de Stress (ISSL), os quais apontam $56 \%$ de pessoas com estresse na população geral (Lipp, 2005).

Corbiére et al. (2015) observaram uma série de sintomas como mais apontados por trabalhadores afastados do trabalho por depressão e, em quase sua totalidade, são iguais aos sintomas de estresse presentes no ISSL, assim, nota-se uma aproximação desses dados com os dados da literatura, uma vez que não foram encontrados estudos que analisassem o estresse em trabalhadores afastados.

A distribuição do estresse evidenciado, em função do sexo do participante, revelou uma diferença significativa a 0,01 . Este se manifestou com maior prevalência entre as mulheres, sendo que do total de 68 mulheres $77,9 \%$ o apresenta, e de 48 homens, 56,3\%. A literatura especializada traz essa diferença como algo constante e evidente nos estudos que relacionam estresse e gênero (Calais, 2003).

A elevada prevalência de estresse na amostra encontra sustentação em estudos populacionais como de Holden, Scuffham, Hilton, Ware, Vecchio e Whiteford (2011), que afirmam que este tende a ser comórbido a doenças que levam ao absenteísmo e afastamento. Ainda na compreensão desses dados, Carr e Umberson (2013), apontam que o estresse recebe influências de variáveis sociais e Souza et al. (2015) dizem que as pessoas reagem de modo diferente aos estímulos causadores de estresse, em função de fatores individuais. Entendendo a comorbidade aqui como do tipo prognóstica, ou seja, do tipo de quando existe uma doença que predispõe o paciente a desenvolver outras doenças (Marques et al., 1994), pode-se compreender melhor e considerar o estresse como comórbido às doenças que levam ao afastamento do trabalho, pois além de estudos que corroboram isso, observa-se que, sendo o estresse resultado da avaliação cognitiva que o indivíduo faz das exigências ambientais a que está exposto, estando o trabalhador afastado socialmente fragilizado e exposto a uma gama elevada de estressores, o estresse tenderá a aparecer e se instalar.

Os fatores relativos a gênero, idade e facilidade de se recolocar no mercado em função da profissão exercida têm influência no desenvolvimento e manutenção do estresse e parecem, aqui, estarem sendo representadas pela diferença na prevalência de estresse. 
Berger e Luckmann (1990) consideram que a percepção é uma atividade psicológica que deve ser interpretada a partir do contexto psicossocial ao qual o indivíduo que percebe se encontra. Sendo assim, a percepção social compreende um conjunto de valores e crenças que filtram a realidade que circunda o indivíduo, atribuindo-lhe significados.

Tomando os dados obtidos pelo Ipat, relativos à percepção da condição de afastamento do trabalho, observou-se que o grupo de trabalhadores afastados, que apresentaram estresse, percebe de forma significativamente pior as expectativas de recepção no ambiente de trabalho ao retornarem $(\mathrm{F} 1 \mathrm{p}=0,01)$, o suporte social à condição de afastamento $(\mathrm{F} 2 \mathrm{p}=0,01), \mathrm{o}$ apoio familiar que têm ( $\mathrm{F} 4 \mathrm{p}=0,05)$ e a percepção de sua capacidade para o trabalho ( $F 5 \mathrm{p}=0,02)$, quando comparados ao grupo de trabalhadores afastados que não apresentam estresse. A análise interna das 22 afirmativas do Ipat revelou diferenças significativas nas médias gerais dos grupos sem e com estresse em dez, demostrando uma percepção mais positiva da condição de afastamento pelos trabalhadores afastados e sem estresse. Isso remete ao que pontua St-Arnould et al. (2014) de que há uma grande interferência do estresse nos processos de reinserção do trabalhador afastado ao retornar de sua licença e evidencia a necessidade de se estabelecer programas estruturados de retorno ao trabalho. St-Arnould et al. (2014) apresentam um programa estruturado de retorno ao trabalho, desenvolvido sobretudo no Canadá, no qual entre outras atividades existe um protocolo de contato e preparação da equipe e dos superiores do trabalhador quando este entra em processo de retorno ao trabalho, isto é feito, entre outros objetivos, para reduzir o estresse que interfere na readaptação do trabalhador afastado.

Toldrá, Daldon, Santos e Lancman (2010), analisando os facilitadores e barreiras para o retorno ao trabalho, apontam como fatores facilitadores o apoio e os relacionamentos e, observando os dados obtidos, notam-se diferenças entre os grupos com e sem estresse no que se refere à percepção do apoio familiar, dos colegas de trabalho e chefes. Nesse caso é perceptível que o estresse está interferindo negativamente, porém, dada sua natureza, não é possível apontar se o estresse advém da condição de afastamento ou da falta de apoio percebida.

Neste momento faz-se interessante retornar ao conceito de comorbidade, tomando-a como do tipo prognóstica (Marques et al., 1994). Se a comorbidade prognóstica é a que se manifesta quando existe uma doença que predispõe o paciente a desenvolver outras doenças, em um processo de análise circular temse que o estresse pode estar advindo da condição psicossocial de afastamento e ao mesmo tempo potencializando a doença geradora desse afastamento, gerando um círculo vicioso.

Estudos interdisciplinares sobre as Doenças do Sistema Osteomuscular e do Tecido Conjuntivo, entre elas a LER/Dorts, têm sido realizados tanto no Brasil como no mundo e apontam para uma origem multicausal, envolvendo aspectos não puramente biomecânicos, mas psicológicos e sociais como contribuintes ao seu desenvolvimento (Andrade \& Barbosa-Branco, 2015; Bugajska et al., 2013; Zakerian \& Subramaniam, 2011; Almeida et al., 2008 e National Research Council, 2001). A experiência de reabilitação e reintegração ao trabalho de portadores de LER/Dorts, relatada 
por Lima et al. (2010), evidencia que uma atuação interdisciplinar e interinstitucional, baseada em uma abordagem biopsicossocial, apresenta bons resultados no processo de reabilitação da funcionalidade do trabalhador acometido por esses distúrbios; e os dados, aqui relatados, demonstram a importância de se observar e cuidar do estresse para uma reinserção adequada desses trabalhadores no mercado de trabalho.

\section{Considerações Finais}

O estresse tem se tornado um "vilão" da sociedade moderna e, sobretudo em sua vertente ocupacional, tem se apresentado cada dia mais intenso e prejudicial à população. Dada a necessidade de se discutir adequadamente o tema, objetivou-se aqui tratar do estresse comórbido à condição de "forçoso não trabalho", ou seja, o estresse presente nos casos de afastamento do trabalho por motivo de saúde.

Os dados apontaram uma forte influência negativa do estresse na percepção da condição de afastamento do trabalho, contaminando a percepção de suporte social e da família, percepção do ambiente de trabalho e da própria capacidade para o trabalho. Essa influência pode prejudicar de forma bastante contundente a melhora no quadro de saúde do trabalhador afastado e dificultar sobremaneira a sua reinserção no mercado de trabalho, gerando problemas psicológicos, sociais e econômicos.

Não cabe aqui a discussão se a condição de afastamento gerou o estresse ou o estresse gerou o problema osteomuscular que levou ao afastamento. Observou-se que as diferenças entre grupos com e sem estresse foram marcantes na amostra pesquisada e isso leva a se pensar o quanto a percepção da condição de afastamento está interligada à geração de uma resposta de estresse que, por sua vez, pode agravar o estado de saúde orgânica do indivíduo, interferindo na sua recuperação e reinserção no mercado de trabalho. Assim sendo, torna-se uma condição fundamental olhar para o problema do estresse nessa parcela da população, de modo a garantir uma adequada recuperação da saúde e retorno ao mercado e à vida em sociedade.

Finalmente, faz-se importante pontuar a limitação inerente aos levantamentos quantitativos, pois estes não permitem averiguar a dinâmica de manifestação do sofrimento oriundo da condição de afastamento; sugere-se que novos trabalhos voltem-se à compreensão dessa dinâmica do sofrimento e, também, deem voz e espaço de expressão a essa parcela da população.

\section{Referências}

Almeida, J. S., Carvalho Filho, G., Pastre, C. M., Lamari, N. M., \& Pastre, E. C. (2008). Afecção do tensão supra-renal e afastamento laboral. Ciência \& Saúde Coletiva. 13(2), 517-522. Recuperado em 15 agosto, 2016, de http://www.scielo.br/pdf/csc/v13n2/a 27v13n2.pdf

Andrade, D. M., \& Barbosa-Branco, A. (2015). Sinovite no Brasil: uma análise dos benefícios auxílio-doença. Revista Brasileira de Epidemiologia. 18(1), 194-207. Recuperado em 28 novembro, 2016, de http://www.scielo.br/pdf/rbepid/v18n 1/1415-790X-rbepid-18-01-00194.pdf

Antloga, C. S., Maia, M., Cunha, K. R., \& Peixoto, J. (2014). Contexto de trabalho e custo humano no trabalho em um órgão do poder judiciário brasileiro. Ciência e Saúde 
Coletiva 19(12), 4787-4796. Recuperado em 28 novembro, 2016, de http://www.scielo.br/pdf/csc/v19n12/ 1413-8123-csc-19-12-04787.pdf

Benzoni, P. E. (2012). Afastamento do trabalho: medos e angústias do retorno após o afastamento por DORT em metalúrgicos. In $V$ Congresso Brasileiro de Psicologia Organizacional e do Trabalho. Rio de Janeiro, Brasil. Anais. Associação Brasileira de Psicologia Organizacional e do Trabalho.

Benzoni, P. E., \& Bragion, A. (2016). Construção e Validação do Inventário de Percepção de Afastamento do Trabalho. Revista Trabalho (En)Cena, Palmas-TO, 1(2), 162175. Recuperado de https://sistemas.uft.edu.br/periodicos/i ndex.php/encena/article/view/2607

Berger, P. L., \& Luckmann, T. (1990). A construção social da realidade: tratado de sociologia do conhecimento (F. S. Fernandes, trad.). Petrópolis: Vozes.

Boschco, C. R. (2011). Implicações do afastamento do trabalho por adoecimento na subjetividade do auxiliar de enfermagem. Dissertação de Mestrado, Universidade Federal do Paraná, Curitiba, Brasil.

Bugajska, J., D., Jędryka-Góral, A., Gasik, R., Hildt-Ciupińska, K., Malińska, M., \& Bedyńska, S. (2013). Psychological Factors at work and Musculoskeletal Disorders: A One Year Prospective Study. Rheumatology International (Switzerland), 33(12), 2975-2983. Recuperado em 18 abril, 2016, de http://www.ncbi.nlm.nih.gov/pmc/arti cles/PMC3832752/pdf/296_2013_Arti cle_2843.pdf

Calais, S. L. (2003). Diferenças entre homens e mulheres na vulnerabilidade ao stress. In LIPP, M. E. N.. (Org.). Mecanismos neuropsicofisiológicos do stress: teoria e aplicações clínicas. São Paulo: Casa do Psicólogo, 8789.

Carr, D., \& Umberson, D. (2013). The Social Psychology of Stress, Health, and Coping. Handbook of Social Psychology. Springer.

Corbière, M., Samson, E., Negrini, A., St-Arnaud, L., Durand, M., Coutu, M., Sauvé, G., \& Lecomte, T. (2015). Factors Perceived by Employees Regarding Their Sick Leave Due to Depression. Disability and Rehabilitation, 38(6), 511-519. Recuperado em 17 novembro, 2016, de https://www.ncbi.nlm.nih.gov/pubmed /25974227

Eatough, E. M., Way, J. D, \& Chang, C. (2012). Understanding the Link between Psychosocial Work Stressors and WorkRelated Musculoskeletal Complaints. Applied ergonomics, 43(3), 554-563.

Holden, L., Scuffham, P. A., Hilton M. F., Ware, R. S., Vecchio, N. E., \& Whiteford, H. A. (2011). Health-Related Productivity Losses Increase When the Health Condition is Co-Morbid with Psychological Distress: Findings from a Large Cross-Sectional Sample of Working Australians. BMC Public Health, 417(11). Recuperado em 20 agosto, 2015, de

http:/ /bmcpublichealth.biomedcentral.c om/articles/10.1186/1471-2458-11-417 
Leka, S., Griffiths, A., \& Cox, T. (2004). Work Organization and Stress: Systematic Problem Approaches for Employers, Managers and Trade Union Representatives, 3. OMS. Recuperado em 10 janeiro, 2016, de http://www.who.int/occupational_healt $\mathrm{h} /$ publications/en/oehstress.pdf

Lima, M. A. G., Andrade, A. G. M., Bulcão, C. M. A., Mota, E. M. C. L., Magalhães, F. B., Carvalho, R. C. P., Freitas, S. M. C., Sá, S. R. F. C., Porto, L. A., \& Neves, R. F. (2010). Programa de reabilitação de trabalhadores com LER/DORT do Cesat/Bahia: ativador de mudanças na Saúde do Trabalhador. Rev. bras. Saúde ocup., São Paulo, 35(121), 112-121, Recuperado em 30 novembro, 2016, de http://www.scielo.br/scielo.php?script= sci_arttext\&pid=S0303-

76572010000100012

Lipp, M. E. N. (2005). Manual do inventário de sintomas de stress para adultos de Lipp (ISSL) (3a ed.). São Paulo, SP: Casa do Psicólogo.

Martins, P. F., Nascimento Sobrinho, L. C., Silva, M. V., Pereira, N. B., Gonçalves, C. M., Rebouças, B. S., \& Cartaxo, L. A. (2009). Afastamento por doença entre trabalhadores de saúde em um hospital público do estado da Bahia. Revista Brasileira de Saúde Ocupacional (São Paulo), 34(120), 172-178. Recuperado em 6 outubro, 2016, de http://www.scielo.br/pdf/rbso/v34n12 0/08v34n120.pdf

Martinez, M. C., Latorre, M. R. D. O., \& Fischer, F. M. (2010). Capacidade para o trabalho: revisão de literatura. Ciência \& Saúde
Coletiva, 15(1), 1553-1561. Recuperado em 30 abril, 2016, de http://www.scielo.br/pdf/csc/v15s1/0 67.pdf

Marques, C., Nardi, A. E., Figueira, I., Mendlowicz, M., Andrade, Y., Camisão, C. Versiani, M. (1994). Comorbidade: conceito e implicações na pesquisa clínica em psiquiatria. J Bras Psiquiatr, 3(43), 117-121. Recuperado em 16 agosto, 2017, de http://webcache.googleusercontent.co m/search?q=cache:http://pesquisa.bvs. $\mathrm{br} / \mathrm{brasil} /$ resource/pt/psi2670\&gws_rd=cr\&ei=rkWXWaz7J4Oh wATPiqeICg

Ministério da Previdência Social/Brasil. (2014). Anuário estatístico da Previdência Social. Brasília: MPS/Dataprev. Recuperado em 15 setembro, 2016, de http://www.previdencia.gov.br/wpcontent/uploads/2016/07/AEPS2014.pdf

Ministério do Trabalho e Emprego/Brasil. (2010). Classificação Brasileira de Ocupações. Brasilia, DF: MTP/SPPE. Recuperado em 10 setembro, 2016, de http://www.mtecbo.gov.br/cbosite/pag es/home.jsf

National Research Council and Institute of Medicine/EUA. (2001). Musculoskeletal disorders and the workplace: low back and upper extremities. Washington, DC: The National Academies Press.

Ramos, M. Z., Tittoni, J., \& Nardi, H. C. (2008). A experiência de afastamento do trabalho por adoecimento vivenciada como processo de ruptura ou 
continuidade nos modos de viver. Cadernos de Psicologia do Trabalho, 11(2), 209-216. Recuperado em 20 setembro, 2016, de http://www.revistas.usp.br/cpst/article /view/25781/27514

Selye, H. (1950). Stress and the General Adaptation Syndrome. British Medical Journal, 1(4667).

Selye, H. (1959). Stress: a tensão da vida. São Paulo: Ibrasa.

Silveira Maissiat, G., Lautert, L., Dal Pai, D., \& Tavares, J. P. (2015). Contexto de trabalho, prazer e sofrimento na atenção básica em saúde. Revista Gaúcha de Enfermagem, 39(2), 42-49. Recuperado em 2 dezembro, 2016, de http://seer.ufrgs.br/index.php/Revista GauchadeEnfermagem/article/view/51 128

Souza, M. E. L., \& Faiman, C. J. S. (2007). Trabalho, saúde e identidade: repercussões do retorno ao trabalho, após afastamento por doença ou acidente, na identidade profissional. Saúde, Ética \& Justiça, 12(1/2), 22-32. Recuperado em 3 novembro, 2016, de http://www.revistas.usp.br/sej/article/v iew/44356/47977

Sousa, M. B. C., Silva, H. P. A., \& Galvão-Coelho, N. L. (2015). Resposta ao estresse: I. Homeostase e teoria da alostase. Estudos de Psicologia, 20(1), 2-11. Recuperado em 5 abril, 2016, de http://www.scielo.br/pdf/epsic/v20n1 /1413-294X-epsic-20-01-0002.pdf

St-Arnaud, L., Briand, C., Corbière, M., Durand, M., Bourbonnais, R., Saint-Jean, M.,
Pelletier, M., Delisle, S., \& Kedl, E. (2014). Supporting a Return to Work After an Absence for a Mental Health Problem: Design, Implementation, and Evaluation of an Integrated Practices Program, Montréal (CA). IRSST Communications and Knowledges.

Toldrá, R, C., Daldon, M. T. B., Santos, M. C., \& Lancman S. (2010). Facilitadores e barreiras para o retorno ao trabalho: a experiência de trabalhadores atendidos em um Centro de Referência em Saúde do Trabalhador - SP, Brasil. Revista Brasileira de Saúde Ocupacional, 35(121), 1022. Recuperado em 10 julho, 2014, de http://www.scielo.br/scielo.php?script= sci_arttext\&pid=S0303-

76572010000100003

Zakerian, S. A., \& Subramaniam, I. D. (2011). Examining the Relationship between Psychosocial Work Factors and Musculoskeletal Discomfort among Computer User in Malaysia. Iranian Journal of Public Health (Iran), 40(1), 7279. Recuperado em 10 janeiro, 2016, de https://www.ncbi.nlm.nih.gov/pmc/art icles/PMC3481727/

Recebido em: 06/05/2017

Aprovado em: 03/02/2018 